\title{
The Composition and Phosphate-Solubilizing Capability of Phosphate-Solubilizing Bacteria in the Rhizosphere of Wild Camellia oleifera in Mountain Lushan
}

\author{
Qibiao Sun ${ }^{1, \dagger}$, Yanfen Liu ${ }^{1, \dagger}$, Yan Tang ${ }^{1}$, Peiyu Zhang ${ }^{1}$, Yao Tong ${ }^{1}$, Gang He $^{1}$, Xiaohong Ji $^{1}$, \\ Zhenying $\mathrm{He}^{2}$, Jianping Ouyang ${ }^{1}$, Hongfang Zhang ${ }^{1, *}$, Ye Chen ${ }^{1, *}$ \\ ${ }^{1}$ College of Pharmacy and Life Sciences, Jiujiang University, Jiujiang, China \\ ${ }^{2}$ College of Electronic Commerce, Jiujiang University, Jiujiang, China
}

\section{Email address:}

sunqibiao001@163.com (Qibiao Su),3138803064@qq.com (Yanfen Liu),2429170761@qq.com (Yan Tang), 2024525323@qq.com (Peiyu Zhang),1165326672@qq.com (Yao Tong), ganghe_myc@126.com (Gang He),

xhji2010@163.com (Xiaohong Ji),1187099020@qq.com (Zhenying He), 86956564@qq.com (Jianping Ouyang), 939044612@qq.com (Hongfang Zhang), chenyejjtc@126.com (Ye Chen)

${ }^{*}$ Corresponding author

$\uparrow$ Qibiao Sun and Yanfen Liu are co-first authors.

\section{To cite this article:}

Qibiao Sun, Yanfen Liu, Yan Tang, Peiyu Zhang, Yao Tong, Gang He, Xiaohong Ji, Zhenying He, Jianping Ouyang, Hongfang Zhang, Ye Chen. The Composition and Phosphate-Solubilizing Capability of Phosphate-Solubilizing Bacteria in the Rhizosphere of Wild Camellia oleifera in Mountain Lushan. American Journal of Agriculture and Forestry. Vol. 9, No. 3, 2021, pp. 141-146. doi: 10.11648/j.ajaf.20210903.17

Received: May 14, 2021; Accepted: May 26, 2021; Published: May 31, 2021

\begin{abstract}
Camellia oleifera, a unique edible oil tree species in China, is of important economic value. However, the shortage of phosphorus in the soil is one of the important factors limiting the growth of C. oleifera. Here, we investigated the population size and composition of culturable phosphate-solubilizing bacteria (PSB) in the rhizosphere soil of wild C. oleifera in Mountain Lushan, China. PSB were isolated using a dilution coating plate method and identified by 16S rRNA sequencing. The phosphate-solubilizing capability of the isolated PSB was evaluated by a semi-quantitative method (the ratio of phosphate solubilization halo diameter versus colony diameter). The results showed that large amounts of PSB existed in the rhizosphere soil of wild C. oleifera $\left(0.28-1.08 \times 10^{7} \mathrm{CFU} / \mathrm{g}\right.$ soil $)$ and the population size of PSB differed from investigated trees. A total of 100 strains of PSB were isolated from the rhizosphere soil, belonging to Bacillus, Burkholderia, Pantoea, Paraburkholderia, and Pseudomonas, respectively. Of these strains, Burkholderia showed the highest isolation frequency and phosphate-solubilizing capability, accounting for $61 \%$ of the isolates. The phosphate solubilization index of 100 strains varied from 1.02 to 3.04 after a 6-day incubation, and Bacillus strains were easy to lose their phosphate-solubilizing capability during the incubation. Our result suggested that Burkholderia was the dominant genus of PSB in the rhizosphere of C. oleifera and could be utilized for facilitating the uptake of $P$.
\end{abstract}

Keywords: Camellia oleifera, Phosphate-solubilizing Bacteria, 16S rRNA, Phosphate Solubilization Index

\section{Introduction}

Phosphorus $(\mathrm{P})$ is one of the most important macronutrients for the basic metabolic and physiological processes in plants including photosynthesis, energy transfer, signal transduction, macromolecular biosynthesis, and respiration, etc. [1]. Although the total $\mathrm{P}$ content is high in soils, the majority is in insoluble forms difficult to be absorbed by plants. Soluble inorganic phosphate $(\mathrm{Pi})$ is the only form available to plants directly, but its concentration in the soil is very low, usually 1$10 \mu \mathrm{M}$, only accounting for about $5 \%$ of total $\mathrm{P}$ in the soil [2].

Camellia oleifera (Theaceae), a unique edible oil tree species in China, is one of the world's famous woody oil plants [3]. Tea oil obtained from the seeds has oleic acid and linoleic acid-based unsaturated fatty acid contents up to $90 \%$, along with large quantities of vitamin $\mathrm{E}$, squalene, and flavonoid substances that are reported to have good health 
care effect on digestion, immunity, reproduction, heart and cerebrovascular, etc., known as "Oriental olive oil" [4]. However, available Pi content in the soil of C. oleifera is often in a state of shortage, which is an important factor limiting the growth of $C$. oleifera $[5,6]$. There are a large number of microorganisms in the soil, which can transform $\mathrm{P}$ resource that is difficultly accessed by plants into a form that can be absorbed and utilized. Solubilization of insoluble $\mathrm{Pi}$ by microorganisms was firstly reported by Pikovskaya [7]. Phosphate-solubilizing microorganisms are important microbial resources closely related to plant nutrition, accounting for about $10 \%$ of the total soil microorganisms [8]. At present, 44 genera of phosphate-solubilizing bacteria (PSB) have been reported, of which Actinomyces, Bacillus, Burkholderia, Pseudomonas, and Streptomyces are the most common taxa [9-14]. In recent years, studies showed that there are larges of PSB taxa in the rhizosphere of C. oleifera [14-17], suggesting the enrichment of PSB in the rhizosphere may be one of the important mechanisms for $C$. oleifera adapting to low P stress. For example, Bacillus aryabhattai JX285 and Pseudomonas auricularis HN038 isolated from the rhizosphere of $C$. oleifera can significantly increase the available $\mathrm{P}$ content in the rhizosphere and the total $\mathrm{P}$ content in C. oleifera trees [18]. The insoluble $\mathrm{Pi}$ in soils is mainly divided into two types: inorganic $\mathrm{Pi}$ and organic $\mathrm{Pi}$, and the insoluble inorganic Pi is dominant [19]. However, previous studies on rhizospheric PSB of $C$. oleifera mainly focused on their biodegradation of organic phosphorus [15-17], but the phosphate-solubilizing capability on insoluble Pi by PSB and their taxonomic composition in the rhizosphere of $C$. oleifera are still unclear. Therefore, this study aimed to study the composition of PSB in the rhizosphere of $C$. oleifera based on a culture-dependent method and analyzed their differences in phosphate-solubilizing capability, which will be conducive to mining PSB resource to promote the growth of $C$. oleifera.

In this study, we determined the population size of PSB in the rhizosphere soil of wild $C$. oleifera in Mountain Lushan, Jiangxi Province, China. PSB were isolated using a plating method and identified by $16 \mathrm{~S}$ rRNA sequencing. The phosphate-solubilizing capability of the isolated PSB was evaluated by a semi-quantitative method. We aimed to reveal the community composition of PSB in the rhizosphere soil of $C$. oleifera based on a culture-dependent method, assess their phosphate-solubilizing capability, and screen potential efficient PSB.

\section{Materials and Methods}

\subsection{Study Site and Sample Collection}

The study site is located in Mountain Lushan, Jiangxi province, China, where the annual average temperature is $11.4^{\circ} \mathrm{C}$ and the annual average rainfall is $1,916 \mathrm{~mm}$. Rhizosphere soil samples of wild C. oleifera were collected at Sanbaoshu scenic area in September 2020. This area is an evergreen and deciduous broad-leaved mixed forest with an altitude of about $900 \mathrm{~m}$. Five $C$. oleifera trees $(\mathrm{CO} 1, \mathrm{CO} 2$, $\mathrm{CO} 3, \mathrm{CO} 4$, and $\mathrm{CO} 5$ ) were chosen and each other was over 10 $\mathrm{m}$ apart. Roots of $C$. oleifera were collected and the soil attached to the root was taken as rhizosphere soil after removing bulk soil by slight shaking. Then, rhizosphere soil was obtained and collected by ultrasonic cleaning in sterilized physiological saline solution $(0.85 \%)$ for $10 \mathrm{~min}$ and centrifuging at 10,000 rpm for $5 \mathrm{~min}$.

\subsection{Population Size of PSB in the Rhizosphere of $C$. oleifera}

Five-gram rhizosphere soil was floated in $50 \mathrm{ml}$ sterilized physiological saline solution. An aliquot $(100 \mu \mathrm{l})$ from $10^{4}$, $10^{5}$ and $10^{6}$ dilution was respectively inoculated on NBRIP agar media (Glucose $10 \mathrm{~g}, \mathrm{NaCl} 0.2 \mathrm{~g}, \mathrm{KCl} 0.2 \mathrm{~g},\left(\mathrm{NH}_{4}\right)_{2} \mathrm{SO}_{4}$ $0.5 \mathrm{~g}, \mathrm{MgSO}_{4} \cdot 7 \mathrm{H}_{2} \mathrm{O} 0.3 \mathrm{~g}, \mathrm{MnSO}_{4} 0.03 \mathrm{~g}, \mathrm{FeSO}_{4} \cdot 7 \mathrm{H}_{2} \mathrm{O} 0.01 \mathrm{~g}$, tricalcium phosphate $5 \mathrm{~g}$, agar $15 \mathrm{~g}, \mathrm{pH} 7.0$ ), and incubated at $30{ }^{\circ} \mathrm{C}$ for three days. Each dilution was in triplicate. After incubation, the colony-forming units (CFU) of bacteria with solubilization halos were counted.

\subsection{Isolation, Purification and Identification of PSB}

Colonies showing large clear halo zone of phosphate solubilization appearing on agar media were streaked on fresh NBRIP agar media for purification and finally transferred in NBRIP agar slants and stored at a $4{ }^{\circ} \mathrm{C}$ refrigerator for further studies. A total of 20 PSB strains were randomly selected from each rhizosphere of $C$. oleifera. The selected isolates were respectively incubated in Luria-Bertani (LB) liquid media at $30{ }^{\circ} \mathrm{C}$ and $180 \mathrm{rpm}$ for two days for proliferation. Then, genomic DNA of isolates was extracted using Rapid Bacterial Genomic DNA Isolation Kit (Sangon Biotech, China) according to the manufacturer's instruction. The amplification of $16 \mathrm{~S}$ rRNA referred to the method of Sun et al. [20]. The amplified products were sent for Sanger sequencing at Sangon Biotech, China. Sequences were analyzed by the BLAST algorithm for comparison of a nucleotide query sequence against GenBank of NCBI to find the closely related sequences. Phylogenetic relationships of selected PSB were constructed using the Maximum Likelihood method in MEGA 7.0 software [21].

\subsection{Phosphate-solubilizing Capability Assay of PSB}

Each strain was incubated in LB liquid medium for two days at $30{ }^{\circ} \mathrm{C}$ and $180 \mathrm{rpm}$. Then, the phosphate-solubilizing capability of PSB was evaluated after inoculating $4 \mu \mathrm{l}$ of inocula on NBRIP agar media at $30^{\circ} \mathrm{C}$ for six days. The growth and diameter of solubilization halos were measured from the $2^{\text {nd }}$ day to the $6^{\text {th }}$ day. The phosphate-solubilizing capability was evaluated by phosphate solubilization index (PSI) that was calculated according to the following formula [22]:

$$
\text { PSI }=\frac{\text { Colony diameter }+ \text { Halo zone diameter }}{\text { Colony diameter }}
$$

\subsection{Statistical Analysis}

All the results were statistically analyzed using IBM SPSS Statistics 20 Software. A comparison between treatments was 
performed using one-way analysis of variance (ANOVA) identified by Tukey's HSD test at $p<0.05$. The heatmap was built using the pheatmap package in $\mathrm{R}$ 3.6.3 software [23]. Data are expressed as mean \pm standard deviation.

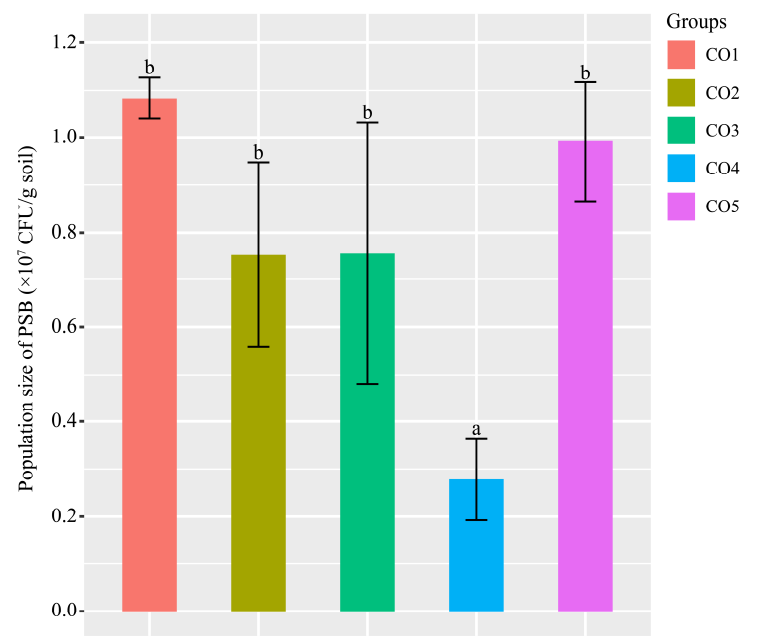

Figure 1. Population size of phosphate-solubilizing bacteria (PSB) in different rhizospheres of $\mathrm{C}$. oleifera. $\mathrm{CO} 1, \mathrm{CO} 2, \mathrm{CO} 3, \mathrm{CO} 4$, and $\mathrm{CO} 5$ donate different rhizosphere soil samples of C. oleifera, respectively.

\section{Results}

\subsection{Population Size of PSB in the Rhizosphere of $C$. oleifera}

The population size of culturable PSB in the rhizosphere of

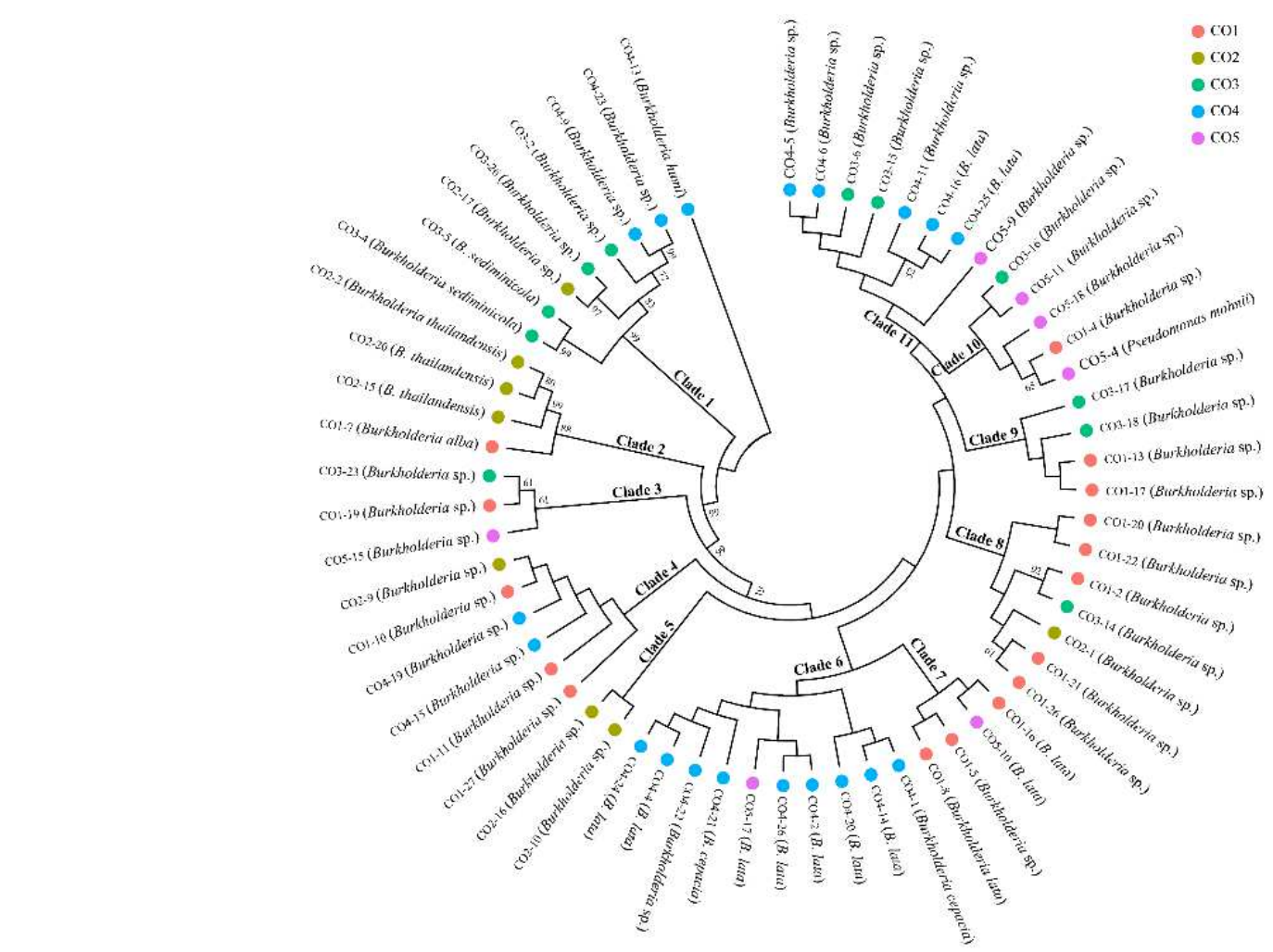

Figure 2. Phylogenetic tree of Burkholderia strains isolated from the rhizosphere of C. oleifera inferred from $16 S$ rRNA sequences. Molecular phylogenetic analysis was using the Maximum Likelihood (ML) method based on the Tamura-Nei model conducted in MEGA7. The discrete Gamma distribution was used to model evolutionary rate differences among sites. Significant bootstrap support (>50) is indicated above branches.
C. oleifera in Mountain Lushan was evaluated by counting the number of phosphate solubilization halos on NBRIP agar media. There was no notable difference in the population size of PSB in different $C$. oleifera rhizospheres. The number of PSB in rhizospheres of five C. oleifera trees was $0.77 \times 10^{7} \pm$ $0.31 \times 10^{7} \mathrm{CFU} / \mathrm{g}$ soil. Except for sample $\mathrm{CO} 4$, the number of PSB in its rhizosphere was $0.28 \times 10^{7} \pm 0.09 \times 10^{7} \mathrm{CFU} / \mathrm{g}$ soil, significantly lower than that in other rhizospheres $(p<0.05$, Figure 1).

\subsection{Taxonomic Composition and Phylogenetic Relationships of PSB}

A total of 100 strains were isolated from the rhizosphere soil of five C. oleifera trees, belonging to Burkholderia, Pantoea, and Pseudomonas in Proteobacteria and Bacillus in Firmicutes. Burkholderia showed the highest isolation frequency (the number of Burkholderia strains versus the total number of isolated strains), accounting for $61 \%$ of the obtained isolates, followed by Bacillus (17\%) and Pseudomonas (14\%). Detailly, Burkholderia accounted for $80 \%$ of the strains isolated from the rhizosphere soil of CO1, $40 \%$ from $\mathrm{CO} 2,55 \%$ from $\mathrm{CO} 3,95 \%$ from $\mathrm{CO} 4$, and $35 \%$ from $\mathrm{CO} 5$, respectively. In addition, the result of phylogenetic analysis of the isolated Burkholderia strains showed that there was no significant difference in the genetic relationships among Burkholderia strains from different rhizosphere soils of $C$. oleifera, except that strains in $\mathrm{CO} 5$ could be clustered into two main clades (Clade 6 and Clade 11, Figure 2). 


\subsection{Difference in the Phosphate-solubilizing Capability of PSB}

A point inoculation method was used to compare the phosphate-solubilizing capability of PSB isolated different rhizospheres of C. oleifera. Figure 3 showed diverse colonial types and phosphate-solubilizing halos formed on NBRIP agar media by PSB isolated from rhizospheres of samples CO1. The values of PSI of the 100 stains from five different rhizospheres of $C$. oleifera were analyzed as shown in Figure 4. Most halo diameters were increased gradually in the period of incubation but differed from different strains. The top five strains with the strongest phosphate-solubilizing capability were CO1-5 (Burkholderia sp., PSI $=3.05 \pm 0.13$ ), CO5-11 (Burkholderia sp., PSI $=2.45 \pm 0.05$ ), CO1-2 (Burkholderia sp., PSI $=2.36 \pm 0.09$ ), $\mathrm{CO} 1-13$ (Burkholderia sp., $\mathrm{PSI}=2.38 \pm$ 0.26), CO5-9 (Burkholderia sp., PSI $=2.26 \pm 0.02$ ), respectively. The results also showed that six strains (Bacillus species) lost phosphate-solubilizing capability or form no halo during the incubation.

\section{Discussion}

Phosphorus is a vitally important mineral nutrient for the growth and reproduction of $C$. oleifera, but the available $\mathrm{P}$ content in the soil where $C$. oleifera trees grow is usually in deficient [5]. Fortunately, large amounts of beneficial rhizobacteria exist in soils, such as PSB that can promote the mobilization of insoluble Pi. In this study, we found many PSB existed in the rhizosphere soil of wild $C$. oleifera trees $\left(0.28-1.08 \times 10^{7} \mathrm{CFU} / \mathrm{g}\right.$ soil, Figure 1$)$, suggesting that may play an important role in the plant against $\mathrm{P}$ deficiency. A total of 100 strains of PSB were isolated from the rhizosphere soil using NIRBP agar media. The strains were identified by $16 \mathrm{~S}$ rRNA sequencing and belonged to four genera (Bacillus, Burkholderia, Pantoea, and Pseudomonas). The top five strains with the strongest phosphate-solubilizing capability all belonged to Burkholderia (Figure 4). Strain CO1-5 (Burkholderia sp.) showed the highest phosphate-solubilizing capability with a PSI value of $3.05 \pm 0.13$ after 6 days incubation, higher than the report from Sengupta et al. [24] and Yasmin and Bano [25] that tested on Picovskaya's agar media using tricalcium phosphate as the sole $\mathrm{P}$ source. Previous studies reported that the most significant solubilizers of phosphate are mainly belonging to Bacillus and Pseudomonas [12, 26-28]. However, in our study, Burkholderia species were easier to be isolated and showed a high phosphate-solubilizing potential (Figure 4). Burkholderia strains formed no specific clade from the result of phylogenetic analysis (Figure 2), suggesting no specific lineage of Burkholderia PSB existed in the rhizosphere of $C$. oleifera. The average value of PSI of Burkholderia (61 strains) isolated from $C$. oleifera rhizospheres was 1.65, higher than those of Bacillus (17 strains, PSI: 1.26), Paraburkholderia (7 strains, PSI: 1.35), and Pseudomonas (14 strains, PSI: 1.25). The results indicated the selection on PSB by the rhizosphere of C. oleifera may contribute to Pi absorption from soil.

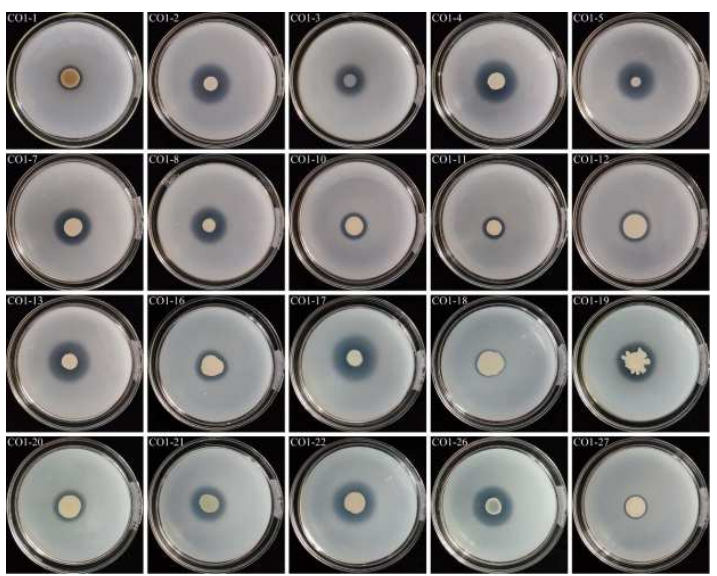

Figure 3. Phosphate-solubilizing halos formed on NBRIP agar media using the point inoculation method by PSB isolated from sample CO1.

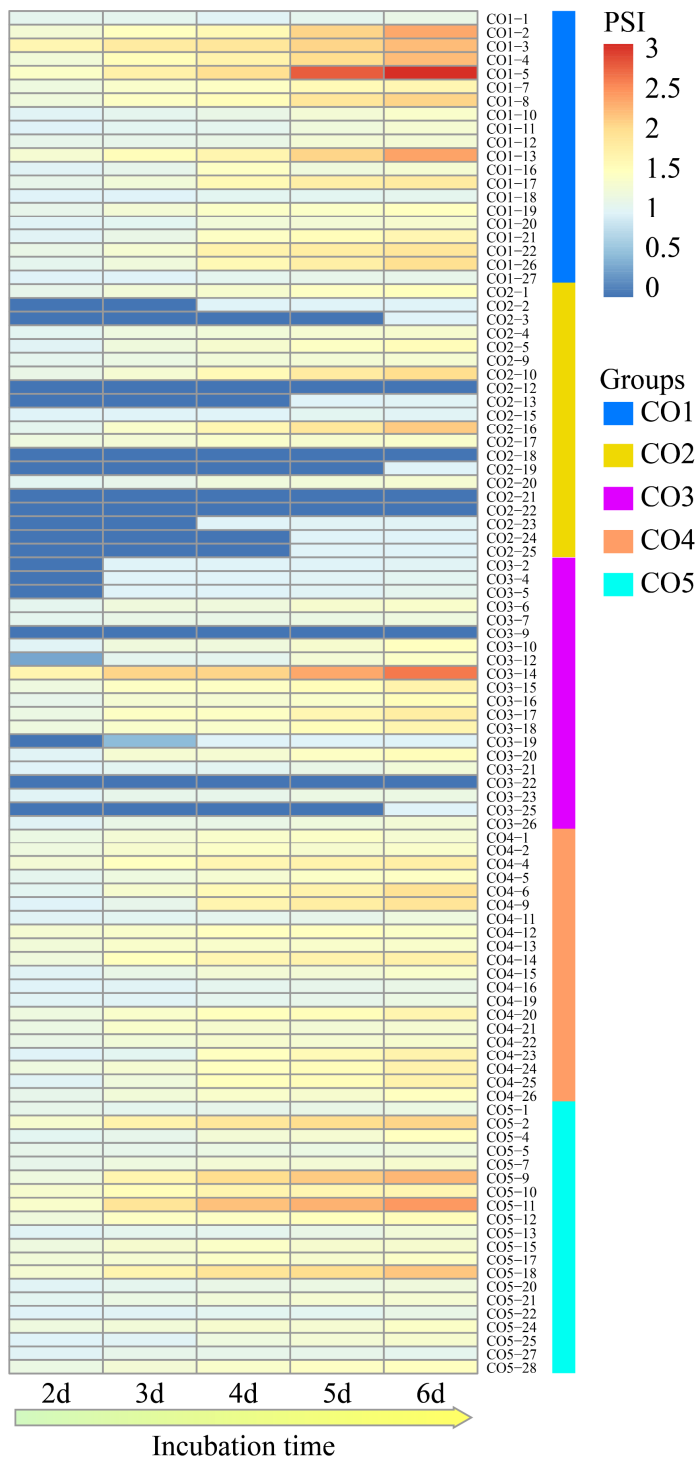

Figure 4. Heatmap of phosphate solubilization index (PSI) values of the 100 PSB isolated from rhizospheres of C. oleifera. 
Although Burkholderia strains had a strong phosphate-solubilizing capability and were unevenly distributed in rhizospheres (Figure 4), there was no significant difference in the overall phosphate-solubilizing potential of PSB in different $C$. oleifera rhizospheres (Figure 5), suggesting that wild $C$. oleifera trees can adjust PSB composition to maintain fair phosphate solubilization. Despite C. oleifera contains Pi transporters that can efficiently absorb soluble Pi in the root-soil interface [29], the soluble Pi cannot meet the requirement of plant growth due to its low concentration in the soil. Plant roots can secrete plant-derived metabolites to affect the composition of rhizosphere bacteria and promote $\mathrm{Pi}$ acquisition [30]. The regulation of rhizosphere bacteria may be one of the important mechanisms for $C$. oleifera adapting to low $\mathrm{P}$ stress. $C$. oleifera usually forms mycorrhiza with arbuscular mycorrhizal fungi (AMF), the fungal symbionts can promote their nutrient absorption [31].

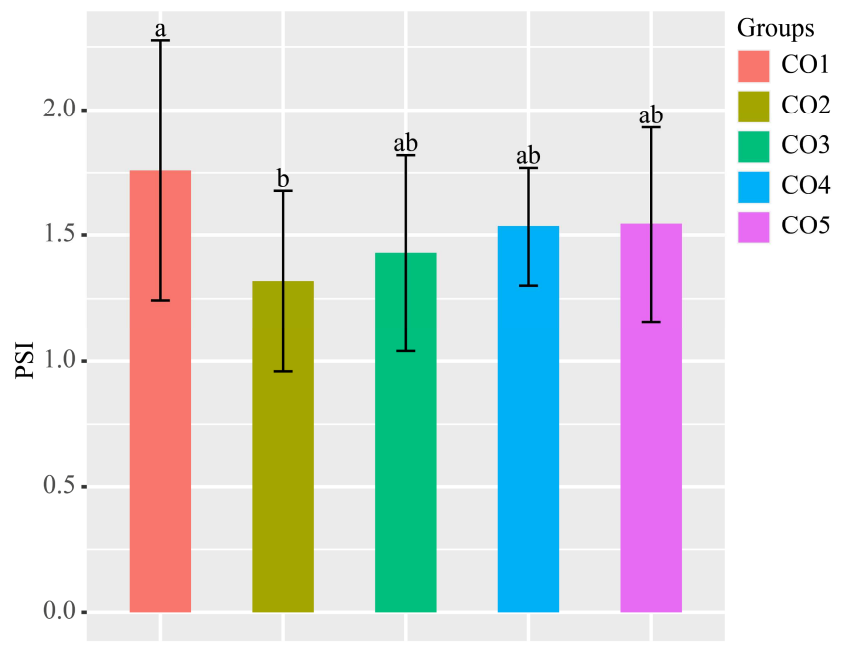

Figure 5. Difference of average phosphate-solubilizing ability of PSB from different rhizospheres.

AMF can promote host plant nutrition by hyphal uptake or regulating beneficial bacteria in the rhizosphere [32-34]. For example, Emmett et al. [35] found that AMF can selectively enrich members of the Proteobacteria. Studies based on culture-dependent methods found that PSB in the rhizosphere of C. oleifera were mainly Burkholderia, Enterobacter, and Pseudomonas in Proteobacteria [15, 17, 18]. In this study, similar results were obtained that strains isolated from the rhizosphere of $C$. oleifera were mainly Burkholderia, Paraburkholderia, Pantoea, and Pseudomonas, indicating that the rhizosphere of $C$. oleifera may enable to select members of Proteobacteria to facilitate the mobilization of insoluble Pi. The phosphate-solubilizing capability of PSB is unstable, easily weakened, or lost during rescreening or incubation $[17,36]$. We also found a similar phenomenon that some strains lost their phosphate-solubilizing capability during incubation, all of which were Bacillus, indicating that the capability of Bacillus strains from the rhizosphere of $C$. oleifera was unstable.

\section{Conclusions}

This study showed a large population size of PSB existed in the rhizosphere of $C$. oleifera. A total of 100 strains of PSB isolated from rhizosphere soils belonged to Bacillus, Burkholderia, Pantoea, Paraburkholderia, and Pseudomonas, of which Burkholderia isolates were the predominant culturable group and shown higher phosphate-solubilizing capability. Of the isolated PSB, Bacillus species had the lower phosphate-solubilizing capability and were easy to lose the capability. Our result suggested that Burkholderia microbes were the efficient PSB resource that can be utilized for facilitating the uptake of $\mathrm{P}$ by $C$. oleifera. In future study, more work should be performed on selecting efficient phosphate-solubilizing isolates for pot experiments to verify their capacity and stability in promoting the absorption of $\mathrm{P}$ by C. oleifera.

\section{Acknowledgements}

This work was found by the National Natural Science Foundation of China (Grant No. 32060010, 31900018), Jiangxi Provincial Natural Science Foundation (Grant No. 20202BAB213025), Research Project of Jiangxi Provincial Science and Technology Department (Grant No. GJJ190925, GJJ190912, GJJ190940) and National Undergraduate Innovation and Entrepreneurship Training Program Project (Grant No. 202011843013).

\section{References}

[1] Khan, M. S., Zaidi, A., Ahemad, M., Oves, M., \& Wani, P. A. 2010. Plant growth promotion by phosphate solubilizing fungi - current perspective. Arch. Agron. Soil Sci., 56, 73-98.

[2] Vance, C., Uhde-Stone, C., \& Allan, D. L. 2003. Phosphorus acquisition and use: critical adaptations by plants for securing a nonrenewable resource. New Phytol., 157, 423-447.

[3] Tan, X. F., Ma, L. Y., Li, D. F., Yao, X. H., Pei, D., Su, S. C., Mao, Y. M., Li, J. A., \& Yuan, D. Y. 2012. Industrialization development strategy of woody grain and oil in China. Nonwood For. Res., 30, 1-5.

[4] Chen, Y. Z., Wang, D. B., \& Su, Y. C. 2001. The fatty acid composition of Camellia oleifera. Nonwood For. Res., 14, 1-4.

[5] Liao, W., Wen, R., Ma, H., Chen, G., Zhang, N., Wang, D., \& Ye, H. 2014. Nutrient elements content in soil and leaf of adult Camellia oleifera forests main areas in central and northern Guangxi. Chinese Journal of Tropical Agriculture, 34, 18-21.

[6] Liu, J., Wu, L., Chen, D., Li, M., \& Wei, C. Soil quality assessment of different Camellia oleifera stands in mid-subtropical China. Appl. Soil Ecol, 2017, 113, 29-35.

[7] Pikovskaya, R. I. 1948. Mobilization of phosphorus in soil in connection with vital activity of some microbial species. Microbiology, 17, 362-370.

[8] Gyaneshwar, P., Kumar, G. N., Parekh, L. J., \& Poole, P. S. 2002. Role of soil microorganisms in improving P nutrition of plants. Plant Soil, 245, 83-93. 
[9] Chen, Y. P., Rekha, P. D., Arun, A. B., Shen, F. T., Lai, W. A., \& Young, C. C. 2006. Phosphate solubilizing bacteria from subtropical soil and their tricalcium phosphate solubilizing abilities. Appl. Soil Ecol., 34, 33-41.

[10] Rodriguez, H., \& Fraga, R. 1999. Phosphate solubilizing bacteria and their role in plant growth promotion. Biotechnol. Adv., 17, 319-339.

[11] Maria, R., Sumera, Y., Mahreen, Y., Claudia, B., Mika, T., \& Thomas, R. 2021. The wheat growth-promoting traits of Ochrobactrum and Pantoea species, responsible for solubilization of different $\mathrm{P}$ sources, are ensured by genes encoding enzymes of multiple P-releasing pathways. Microbiol. Res., 246, 126703.

[12] Sharma, S. B., Sayyed, R. Z., Trivedi, M. H., \& Gobi, T. A. 2013. Phosphate solubilizing microbes: sustainable approach for managing phosphorus deficiency in agricultural soils. SpringerPlus, 2, 587.

[13] Boubekri, K., Soumare, A., Mardad, I., Lyamlouli, K., Hafidi, M., Ouhdouch, Y., \& Kouisni, L. 2021. The screening of potassium- and phosphate-solubilizing Actinobacteria and the assessment of their ability to promote wheat growth parameters. Microorganisms, 9, 470.

[14] Wang, S., Zhang, L. P., Zhang, Y., Hao, F. F., Xiao, J. X., \& $\mathrm{Hu}$ D. N. 2015. Screening, identification and phosphate solubilizing capability of phosphate solubilizing bacteria in rhizosphere of Camellia oleifera Abel at red soil region. Forest Res., 28, 409-416.

[15] Liu, X., Fu, D., Chen, L., Yang, W., Li, D., \& Fu, H. 2015. Isolation, identification and phosphate-solubilizing capacity of phosphate-solubilizing bacteria from the rhizosphere of Camellia. Biotechnology Bulletin, 31, 169-173.

[16] Liu, X., Fu, D., Jia, C., \& Chen, Q. 2016. Isolation, identification and culture condition of phosphate-solubilizing bacteria derived from Camellia rhizosphere soil. Southwest China Journal of Agricultural Sciences, 29, 2637-2642.

[17] Chen, D. A., Wei, X. W., Zhang, M., Cheng, W., Wang, Y. S., Li, Y. L., Wu, SD., \& Yi, H. W. 2020. Isolation, identification and phosphate solubilizing capacity of organophosphorus solubilizing bacteria in rhizosphere soil of Camellia oleifera. Agricultural Science \& Technology, 21, 41-47.

[18] Wu, F., Li, J., Chen, Y., Zhang, L., Zhang, Y., Wang, S., Shi, X., Li, L., \& Liang, J. 2019. Effects of phosphate solubilizing bacteria on the growth, photosynthesis, and nutrient uptake of Camellia oleifera Abel. Forests, 10, 348.

[19] Tang, Y., Lu, L., Yang, Q. Y., \& Yu, G. H. 2006. Application and research progress of phosphate-solubilizing microorganisms. Tianjin Agricultural Sciences, 7, 1-5.

[20] Sun, Q., Li, J., Finlay, R. D., \& Lian, B. 2019. Oxalotrophic bacterial assemblages in the ectomycorrhizosphere of forest trees and their effects on oxalate degradation and carbon fixation potential. Chem. Geol., 514, 54-64.

[21] Kumar, S., Stecher, G., \& Tamura, K. 2016. MEGA7: Molecular Evolutionary Genetics Analysis version 7.0 for bigger datasets. Mol. Biol. Evol., 33, 1870-1874.

[22] Edi Premono, M., Moawad, A. M., \& Vlek, P. L. G. 1996.
Effect of phosphate-solubilizing Pseudomonas putida on the growth of maize and its survival in the rhizosphere. Indonesian J. Crop Sci., 11, 13-23.

[23] R Core Team. 2020. R: A language and environment for statistical computing. R Foundation for Statistical Computing, Vienna, Austria. URL https://www.R-project.org/.

[24] Sengupta, A., Gunri, S. K., Biswas, T., \& Saha, J. 2018. Efficacies of freshly isolated phosphate solubilising bacteria (PSB) on growth promotion in groundnut (Arachis hypogaea L.) upon commonly used PSB biofertilizers in eastern India. Legume Res., LR-4020, 1-7.

[25] Yasmin, H., \& Bano, A. 2011. Isolation and characterization of phosphate-solubilizing bacteria from rhizosphere soil of weeds of Khewra salt range and Attock. Pak. J. Bot., 43, $1663-1668$.

[26] Kucey, R. M. N., Janzen, H. H., \& Legget, M. E. 1989. Microbial mediated increases in plant-available phosphorus. Adv. Agron., 42, 199-228.

[27] Paul, D., \& Sinha, S. N. 2017. Isolation and characterization of phosphate solubilizing bacterium Pseudomonas aeruginosa KUPSB12 with antibacterial potential from River Ganga, India. Annals of Agricultural Sciences, 15, 130.

[28] Mohamed, E. A. H., Farag, A. G., \& Youssef, S. A. 2018. Phosphate solubilization by Bacillus subtilis and Serratia marcescens isolated from tomato plant rhizosphere. J. Environ. Prot., 9, 266-277.

[29] Zhou, J., Lu, M., Zhang, C., Qu, X., Liu, Y., Yang, J., \& Yuan, J. 2020. Isolation and functional characterisation of the PHT1 gene encoding a high-affinity phosphate transporter in Camellia oleifera. J. Hortic. Sci. Biotech., 95, 553-564.

[30] Hütsch, B. W., Augustin, J., Merbach, W. 2002. Plant rhizodeposition: an important source for carbon turnover in soils. J. Plant Nutr. Soil Sci., 165, 397407.

[31] Wang, D. X., Zhang, N. Y., \& Chen, G. C. 2011. Effects of AM fungi on the growth and drought-resistance of Camellia oleifera. Guangxi Forestry Science, 40, 259-273.

[32] Smith, S. E., \& Read, D. 2008. Mycorrhizal Symbiosis, 3 ed., Publisher: Elsevier Academic Press Inc, Netherlands.

[33] Scheublin T. R., Sander, I. R., Keel, C. K., \& van der Meer, J. R. 2010. Characterisation of microbial communities colonising the hyphal surfaces of arbuscular mycorrhizal fungi. ISME J., 4, 752-763.

[34] Jiang, F., Zhang, L., Zhou J., George, T. S., \& Feng, G. 2021. Arbuscular mycorrhizal fungi enhance mineralisation of organic phosphorus by carrying bacteria along their extraradical hyphae. New Phytol., 230, 304-315.

[35] Emmett, B. D., Lévesque-Tremblay, V., Harrison, M. J. 2021. Conserved and reproducible bacterial communities associate with extraradical hyphae of arbuscular mycorrhizal fungi. ISME J. https://doi.org/10.1038/s41396-021-00920-2.

[36] Venkateswarlu, B., Rao, A. V., \& Raina, P. 1984. Evaluation of phosphorus solubilisation by microorganisms isolated from arid soil. Journal of the Indian Society of Soil Science, 32, 273-277. 